\title{
BMJ Open Determining the optimal dose of reactive balance training after stroke: study protocol for a pilot randomised controlled trial
}

\author{
Avril Mansfield (D) , ${ }^{1,2,3}$ Elizabeth L Inness (D) , ${ }^{1,2}$ Cynthia J Danells, ${ }^{1,2}$ \\ David Jagroop (D) , ${ }^{1}$ Tanvi Bhatt, ${ }^{4}$ Andrew H Huntley (D) ${ }^{1}$
}

To cite: Mansfield A,

Inness EL, Danells CJ, et al.

Determining the optimal

dose of reactive balance

training after stroke: study

protocol for a pilot randomised

controlled trial. BMJ Open

2020;10:e038073. doi:10.1136/

bmjopen-2020-038073

- Prepublication history and additional material for this paper are available online. To view these files, please visit the journal online (http://dx.doi org/10.1136/bmjopen-2020038073).

Received 27 February 2020 Revised 30 April 2020 Accepted 20 July 2020

Check for updates

(c) Author(s) (or their employer(s)) 2020. Re-use permitted under CC BY-NC. No commercial re-use. See rights and permissions. Published by BMJ.

${ }^{1}$ Toronto Rehabilitation Institute - University Health Network, Toronto, Ontario, Canada ${ }^{2}$ Department of Physical Therapy, University of Toronto, Toronto, Ontario, Canada ${ }^{3}$ Evaluative Clinical Sciences, Hurvitz Brain Sciences Research Program, Sunnybrook Research Institute, Toronto, Ontario,

Canada

${ }^{4}$ Department of Physical

Therapy, University of Illinois, Chicago, Illinois, USA

Correspondence to

Dr Avril Mansfield;

avril.mansfield@uhn.ca

\section{ABSTRACT}

Introduction Falls risk poststroke is highest soon after discharge from rehabilitation. Reactive balance training (RBT) aims to improve control of reactions to prevent falling after a loss of balance. In healthy older adults, a single RBT session can lead to lasting improvements in reactive balance control and prevent falls in daily life. While increasing the dose of RBT does not appear to lead to additional benefit for healthy older adults, stroke survivors, who have more severely impaired balance control, may benefit from a higher RBT dose. Our longterm goal is to determine the optimal dose of RBT in people with subacute stroke. This assessor-blinded pilot randomised controlled trial aims to inform the design of a larger trial to address this long-term goal.

Methods and analysis Participants $(n=36)$ will be attending out-patient stroke rehabilitation, and will be randomly allocated to one of three groups: one, three or six RBT sessions. RBT will replace a portion of participants' regular physiotherapy so that the total physical rehabilitation time will be the same for the three groups. Balance and balance confidence will be assessed at: (1) study enrolment; (2) out-patient rehabilitation discharge; and (3) 6 months postdischarge. Participants will report falls and physical activity for 6 months postdischarge. Pilot data will be used to plan the larger trial (ie, sample size estimate using fall rates, and which groups should be included based on between-group trends in pre-to-post training effect sizes for reactive balance control measures). Pilot data will also be used to assess the feasibility of the larger trial (ie, based on the accrual rate, outcome completion rate and feasibility of prescribing specific training doses).

Ethics and dissemination Institutional research ethics approval has been received. Study participants will receive a lay summary of results. We will also publish our findings in a peer-reviewed journal.

Trial registration number NCT04219696; Pre results.

\section{INTRODUCTION}

\section{Background and rationale}

Falls are the most prevalent complications during all stages of stroke recovery. ${ }^{1}$ Along with physical injuries, $88 \%$ of people with stroke who fall develop fear of falling. ${ }^{2}$
Strengths and limitations of this study

- The intervention will replace a portion of participants routine physiotherapy during out-patient rehabilitation. Therefore, the findings will be directly relevant to clinical practice.

- Conversely, there is a risk that patients will decline participation in the study, which requires consent to being randomised to a specific dose of reactive balance training, as they will not want their rehabilitation care to be disrupted.

- This is a pilot study, so it is unlikely that we will be able to make definitive decisions regarding the optimal dose of reactive balance training poststroke.

Falls and fear of falling can lead to inactivity, deconditioning, and lower functional capacity, further increasing fall risk ${ }^{34}$ and reducing quality of life. ${ }^{5}$

Conventional balance training, where the goal is to maintain balance during the balance-challenging exercises, reduces falls in older adults, ${ }^{6}$ but not after stroke. ${ }^{78}$ Reactive balance training (RBT), where clients experience repeated postural perturbations (or loss of balance), ${ }^{910}$ is a novel type of exercise that aims to improve reactive balance control. RBT can prevent falls in older adults and people with Parkinson's disease. ${ }^{11}$ Our nonrandomised study suggests that RBT reduces fall rates after discharge from stroke rehabilitation. ${ }^{12}$ In our previous study, the intervention was implemented as part of routine care, and the dose of RBT depended on client goals and preferences and length of stay, rather than being prescribed by the study protocol. Participants completed 1-12, 30 min RBT sessions (median of six sessions). ${ }^{12}$

Unlike other forms of exercise (eg, resistance training or aerobic exercise), where improvements in physical fitness take weeks or months of regular training, ${ }^{13}$ improved 
reactive balance control with RBT seems to occur with few repetitions, and is maintained for several months without training. Among healthy older adults, just 24 perturbations within a single session of RBT are sufficient to lead to lasting improvements (ie, 6-12 months) in reactive balance control, ${ }^{14}$ and prevent falls in daily life. ${ }^{15}$ One study in people with chronic stroke found that improved reactive balance control with a single session of RBT was retained for 3 weeks post-training. ${ }^{16}$ Almost doubling the dose of RBT does not appear to lead to additional benefit for healthy older adults ${ }^{17}$; however, it is possible that those with stroke would benefit from additional RBT as they have more severely impaired balance than healthy older adults. ${ }^{18}$ While additional training may also promote sustained improvements in reactive balance control beyond 3 weeks, ${ }^{19-21}$ in one study that included people with subacute stroke, reduced fall rates up to 6 months post-training were reported when $29 \%$ of participants completed only one $30 \mathrm{~min}$ session of $\mathrm{RBT}^{12}$ The subacute phase is a crucial period for RBT, due to the high potential for neuroplasticity in this early phase of recovery, ${ }^{22}$ and to the high risk of falls early after stroke. ${ }^{23}$ Therefore, there is a need to establish optimal RBT training parameters in the subacute population with stroke.

\section{Objectives and research questions}

The long-term goal of this work is to determine the optimal dose of RBT in people with subacute stroke. This assessor-blinded pilot randomised controlled trial (RCT) aims to inform the design of a larger trial to address this long-term goal. Specifically, the following questions about the larger trial will be answered with this pilot study:

1. What is the optimal sample size?

2. How long will it take to achieve this sample size?

3. Are the proposed secondary outcome measures feasible?

4. How feasible is it to prescribe a specific dose of RBT to people with subacute stroke within routine out-patient rehabilitation? and

5. What two intervention groups should be included in the larger trial?

\section{Trial design}

The current paper describes the protocol for an assessorblinded pilot RCT (figure 1), following the Standard Protocol Items: Recommendations for Interventional Trial guidelines and checklist. ${ }^{24}$ People who are attending out-patient stroke rehabilitation will be randomly assigned to one of three different doses of RBT. Reactive balance control, functional balance and balance confidence will be measured at study enrolment (within days of admission to out-patient rehabilitation), discharge from out-patient rehabilitation and 6 months postdischarge. Falls in daily life, physical activity and participation will be assessed for 6 months postdischarge.

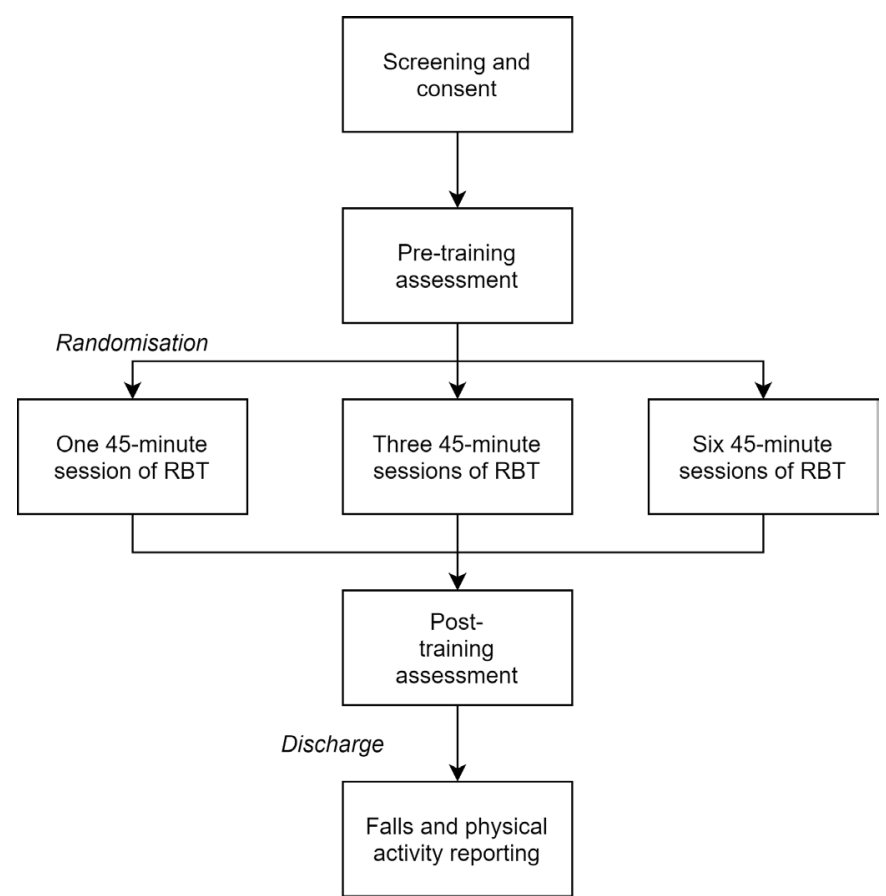

Figure 1 Trial design. RBT, reactive balance training.

Patient and public involvement

This study was designed without patient involvement. Patients were not invited to comment on the study design and were not consulted to develop patient-relevant outcomes. Some trial design elements were informed by participant feedback from our previous RBT study. ${ }^{19}$ Patients were not invited to contribute to the writing or editing of this document for readability or accuracy.

\section{METHODS: PARTICIPANTS, INTERVENTIONS AND OUTCOMES Study setting}

This study will take place at the Toronto Rehabilitation Institute, University Health Network. This facility provides specialised inpatient and out-patient stroke rehabilitation to individuals in the subacute stage of stroke recovery. Out-patient stroke rehabilitation at the Toronto Rehabilitation Institute typically includes $45 \mathrm{~min}$ of physiotherapy 2-5 times/week for at least 4 weeks, with most patients receiving 8 weeks of out-patient rehabilitation.

\section{Participants}

Participants will be people with subacute stroke $(<6$ months poststroke) who are receiving out-patient rehabilitation at the Toronto Rehabilitation Institute. Participants will be eligible if they can: (1) stand independently for $>30 \mathrm{~s}$; (2) walk with or without a gait aid (but without assistance of another person) for $>10 \mathrm{~m}$; and (3) are living in the community. Participants will be excluded if they have:

1. Completed RBT during inpatient rehabilitation.

2. Lower extremity amputation, weight-bearing restrictions, recent lower-extremity injury or surgery (eg, fracture), acute back or lower-limb pain, halo, aspen collar, history of fragility fracture and/or severe osteo- 
porosis/osteopenia, contractures that prevent neutral hip or ankle.

3. Activity restrictions following cardiac event/surgery, abnormal or unstable cardiovascular responses to exercise, arterial dissection.

4. Severe spasticity in the legs that prevents the individual from safely accepting weight on the limb.

5. Cognitive impairment (ie, unable to understand the purpose of training and/or to provide informed consent), as determined by the healthcare team.

6. Acute illness (eg, vomiting, fever), extreme obesity (exceeds safety harness system weight limits), colostomy bags, indwelling catheter, infection, pressure sore on pelvis or trunk.

After participants provide consent, eligibility will be confirmed using information in the participants' hospital chart, by consulting members of the patient's healthcare team, and by consulting the participant themselves. Participants will still receive their usual care while participating in the study.

Participants will be informed that they are free to withdraw from the study at any time point, without consequence. If participants ask to be withdrawn from the study, any data collected from them up to that point will be used to answer the research questions. Participants may also be withdrawn from the study due to changes in their health status that affect eligibility.

\section{Interventions}

Participants will be allocated to one of three groups: one, three or six, 45 min RBT sessions. RBT will replace a portion of participants' regular physiotherapy, so that the total amount of physical rehabilitation will not be affected by study participation, and will be approximately equal for the three groups. Each 45 min session will be entirely dedicated to RBT, and will include up to 60 perturbations. The proposed session duration and number of perturbations per session is double that of our previous subacute study, whereas the number of sessions is halved. ${ }^{12}$ This previous study was conducted during inpatient rehabilitation, where patients are typically provided with $60 \mathrm{~min}$ of physiotherapy 5 days per week. Within this schedule, patients could easily complete $30 \mathrm{~min}$ of RBT, leaving 30 min per day for other physical therapies. However, as out-patient physiotherapy is only $45 \mathrm{~min}$ per session, the proposed dosages more easily fit into most out-patient rehabilitation therapy schedules. From our team's previous research ${ }^{12} 19$ and experience with clinical implementation of RBT in stroke rehabilitation, we expect that participants will be able to tolerate the $45 \mathrm{~min}$ sessions of RBT. Rest breaks will be scheduled into each session, and will be provided when requested by participants.

A research physiotherapist will oversee RBT in collaboration with participants' regular physiotherapists to ensure consistent RBT delivery across participants. Training strategies will be individualised to each participant, based on their balance impairments and rehabilitation goals. ${ }^{12} 19$ For example, if a participant has low foot clearance when executing reactive steps, then obstacles will be placed on the floor and the participant will be encouraged to step over the obstacles during voluntary and reactive stepping. If a participant has a goal to return to a specific activity then aspects of that activity will be included in the training sessions (eg, if returning to golfing is a goal, the participant may train on a compliant surface to simulate uneven outdoor terrain). Further details of the specific balance training approaches that will be used and how training will be tailored to individual participants can be found in our previous paper. ${ }^{19}$ The RBT programme includes multidirectional 'internal' and 'external' balance perturbations. Internal perturbations are achieved by asking the participant to complete tasks that challenge balance control, such that they lose balance when attempting to perform the task (eg, kicking a soccer ball). External perturbations are delivered manually using a push or pull from the physiotherapist while the participant is either standing still or doing a voluntary task, like marching on the spot; when the physiotherapist is positioned behind the participant, the direction and timing of the push or pull can be unpredictable to the participant. As participants improve their reactive balance control, difficulty will be increased by shifting task requirements along a continuum from stable to mobile, and from predictable to unpredictable, and by increasing perturbation magnitude (ie, by increasing the force of the push/pull) or imposing sensory or environmental challenges. ${ }^{25}$

\section{Outcome measures}

To assess feasibility of the study, we will document rates of accrual (ie, number of patients approached to participate in the study vs the number who provide consent), number of training sessions attended/missed, reasons for missed sessions, rate of missing data for the outcomes described below and rate of withdrawal from the study.

Table 1 summarises additional outcome measures. Demographic, stroke information and medical history will be extracted from participants' hospital charts. Participants will complete a questionnaire at baseline that asks about their social supports, employment, familial responsibilities, living situation and so on, which are factors that could influence fall risk. Many of these questions have been adapted from the Canadian Longitudinal Study on Aging. ${ }^{26}$ The National Institutes of Health Stroke Scale ${ }^{27}$ will be scored at study enrolment. Clinical assessments will be scored by a blinded research assistant at three time points: (1) study enrolment (as soon as possible after admission to out-patient rehabilitation); (2) discharge from out-patient rehabilitation; and (3) 6 months postdischarge. Tests will include: Chedoke-McMaster Stroke Assessment ${ }^{28}$ foot and leg scores; mini-Balance Evaluation Systems Test (mini-BEST) ${ }^{29}$; Activities-specific Balance Confidence scale ${ }^{30}$; and reactive balance control following unpredictable and novel perturbations.

To assess reactive balance control, participants will be outfitted with reflective markers, and will complete 8-10 walking trials on a movable platform. There will be four 
Table 1 Cohort descriptors and outcome measures

\begin{tabular}{|c|c|c|c|c|}
\hline & Study enrolment & Discharge & $\begin{array}{l}\text { During 6-month } \\
\text { follow-up }\end{array}$ & $\begin{array}{l}6 \text { Months } \\
\text { postdischarge }\end{array}$ \\
\hline Demographics & $\checkmark$ & & & \\
\hline Time poststroke & $\checkmark$ & & & \\
\hline Lesion location & $\checkmark$ & & & \\
\hline Medical history & $\checkmark$ & & & \\
\hline Medications & $\checkmark$ & & & \\
\hline Changes in health/medications & & $\checkmark$ & & $\checkmark$ \\
\hline $\mathrm{NIH}-\mathrm{SS}^{27}$ & $\checkmark$ & & & \\
\hline $\mathrm{CMSA}^{28}$ & $\checkmark$ & $\checkmark$ & & $\checkmark$ \\
\hline $\operatorname{mini}-B E S T^{29}$ & $\checkmark$ & $\checkmark$ & & $\checkmark$ \\
\hline ABC scale ${ }^{30}$ & $\checkmark$ & $\checkmark$ & & $\checkmark$ \\
\hline Novel unpredictable perturbation & $\checkmark$ & $\checkmark$ & & $\checkmark$ \\
\hline Falls in daily life & & & $\boldsymbol{J}^{*}$ & \\
\hline PASIPD $^{37}$ & & & $\boldsymbol{J}^{*}$ & \\
\hline $\mathrm{SIPSO}^{42}$ & & & $\boldsymbol{J}^{*}$ & \\
\hline
\end{tabular}

*Data collected repeatedly during the 6-month follow-up period.

ABC, Activities-specific Balance Confidence; CMSA, Chedoke-McMaster Stroke Assessment; mini-BEST, mini-Balance Evaluation Systems Test; NIH-SS, National Institutes of Health Stroke Scale; PASIPD, Physical Activity Scale for Individuals with Physical Disabilities; SIPSO,

Subjective Index of Physical and Social Outcome.

force plates embedded in the movable platform. On two trials, the platform will move forward suddenly on heel strike (ie, when one of the force plates is loaded) to trigger a slip-like perturbation. ${ }^{31}$ On two other trials, the platform will move backward suddenly on toe-off (ie, when one of the force plates is unloaded) to trigger a trip-like perturbation. Each slip or trip trial will be triggered on heel-strike or toe-off, respectively, of each of the left and right limbs. The perturbation waveform will consist of a 300 ms square-wave acceleration, followed immediately by $300 \mathrm{~ms}$ deceleration (peak acceleration up to $1.5 \mathrm{~m} / \mathrm{s}^{2}$ ). ${ }^{31}$ The platform will only move during these four trials; the remaining four to six trials will consist of unperturbed walking. The slip/trip and unperturbed walking trials will be presented in a pseudorandom order to ensure that participants cannot predict the timing, direction or perturbed limb for these trials. This unpredictability will help ensure that any changes are not simply due to practice effects on the specific task. While there may be some improvement in responses to the perturbation simply due to repetition of the task (ie, not due to training effects), previous work suggests that experiencing a single slip or trip perturbation does not lead to large and lasting improvement responses to the perturbations. ${ }^{32} 33$ These perturbations differ from what will be used during training, and will measure transfer of training to a novel and ecological loss of balance. Threedimensional motion capture will record the locations of the reflective markers in space. Biomechanical stability when responding to the perturbation will be measured using an established method that considers the distance between the centre of mass and base of support ${ }^{3134}$; in general, a more posteriorly-located (slip) or anteriorlylocated (trip) centre of mass in relation to the perturbed lower limb is considered less stable.

Participants will be asked to report falls ('an event that results in a person coming to rest unintentionally on the ground or other lower level ${ }^{35}$ ) in the 6 months postdischarge. Participants will be provided with stamped, addressed postcards to mail to the research team every 2 weeks for 6 months postdischarge. Postcards will contain a calendar, on which participants will record falls. The blinded research assistant will call participants who do not return the postcard to determine if any falls occurred. The research assistant will contact participants reporting a fall to complete a short questionnaire determining the cause and consequences of the fall. This method is considered the 'gold standard' for fall reporting. ${ }^{36}$

Participants will also report physical activities using the Physical Activity Scale for Individuals with Physical Disabilities (PASIPD), ${ }^{37}$ and participation in daily life using the Subjective Index of Physical and Social Outcome (SIPSO) at 2, 4 and 6 months postdischarge.

\section{Sample size}

We will aim to recruit 12 participants per group (36 participants total), as recommended for pilot studies. ${ }^{38}$

\section{Recruitment}

Participants will be recruited from the Toronto Rehabilitation Institute University Centre out-patient stroke rehabilitation programme. This programme admits 
approximately 200 individuals with stroke per year. Potentially eligible participants will be identified by the patients' primary treating physiotherapist. Participants will be reimbursed for any travel expenses (eg, public transit, taxi or parking) they incur to attend data collection appointments; participants will not be reimbursed for travel expenses for the intervention as they will occur as part of routine care. Participants will also receive a $50 \$ \mathrm{C}$ gift card on completion of the study as a modest incentive to participate.

\section{METHODS: ASSIGNMENT OF INTERVENTIONS Group allocation}

Participants will be assigned using blocked randomisation to one of the three different doses of RBT (block size: $6)$. The random allocation sequence will be computer generated. Blocked randomisation will ensure equal numbers allocated to each group. Group allocation will be performed centrally by the principal investigator, who will not be involved in recruiting, scoring assessments or administering the interventions (ie, concealed allocation).

\section{Blinding}

Outcome measures will be obtained by a research assistant who will be blinded to group allocation. At the discharge and follow-up study visits, the research assistant will be asked to guess the participants' group allocation, and if the research assistant received any information about participant group allocation that led to unblinding. Participants cannot be blinded to group allocation. Data analysis will be conducted by an individual who is not blinded to group allocation.

\section{METHODS: DATA COLLECTION, MANAGEMENT AND ANALYSIS Data collection methods}

Data will be collected primarily by the research assistant either directly from the participant or by chart review (see table 1 for further details). The research assistant has received training regarding data collection from the principal investigator. Questionnaires will be completed via in-person interview at enrolment, and over the telephone at the follow-up time points.

\section{Data management}

Electronic data will be stored on secure institutional servers. Hard copies of files containing de-identified data will be stored in locked cabinets and/or in offices that are locked when not occupied.

\section{Data analysis}

Data analysis will address the research questions as described below.

1. What is the optimal sample size? The proposed primary outcome in the larger trial will be rate of falls in daily life. The one-session group is expected to show minimal improvements in reactive balance control and fall risk. Therefore, the rate of falls (number of falls per person-year) in the one-session group, reported over the 6 months postdischarge, and a clinically meaningful $30 \%$ reduction in fall rates, will be used to estimate sample size for the larger trial. ${ }^{39}$

2. How long will it take to achieve this sample size? We will use the accrual rate (number of participants recruited per month) and proportion of participants who withdraw from the study to estimate how long it will take to achieve the target sample size in the larger trial.

3. Are the proposed secondary outcome measures feasible? Our previous work supports feasibility of data collection using most of the measures in this population. ${ }^{12}$ However, we have not previously tested the slip-like and trip-like perturbations in this population. We will report between-group effect sizes and completeness of data collection for responses to the slip-like and triplike perturbations, and other outcome measures (ie, CMSA, mini-BEST, ABC Scale, PASIPD and SIPSO); the larger trial will only include outcomes with $\geq 80 \%$ completion rate.

4. How feasible is it to prescribe specific dose of RBT to people with subacute stroke? The feasibility of prescribing a specific RBT dose during patients' routine rehabilitation is not known. Participants assigned to the three-session or six-session groups or their physiotherapists may decline sessions if they feel they are not beneficial to their care. Likewise, participants assigned to the one-session or three-session groups or their physiotherapists may feel that they can benefit from additional RBT sessions. The dose will be considered feasible if the mean number of sessions and number of perturbations per session is $75 \%-100 \%$ of prescribed.

5. What two intervention groups should be included in the larger trial? The larger trial will compare one session of RBT with a higher dose. We will use the reactive control subscale of the mini-BEST as a measure of effect of RBT on reactive balance control in each group. Scores on this subscale have been shown to improve with a high dose of RBT in people with chronic stroke. ${ }^{19}$ We will calculate the pre-to-post training effect sizes for this subscale for each group (ie, mean difference in the score from admission to discharge). The minimum detectable change for the total mini-BEST score in people with stroke is 3 points $^{40}$ (ie, $10 \%$ of the maximum score). The minimum detectable change for individual subscales have not been established, but we will assume that this is $10 \%$ of the maximum score for the subscale (ie, 0.6 points). Therefore, if the pre-to-post training effect sizes are within 0.6 points for the three-session and six-session groups, then the larger trial will include the one-session and three-session groups. However, if effect sizes reveal a trend towards greater improvement for the six-session group, then the larger trial will include the one-session and six-session groups.

Data will be analysed at the end of the study. Therefore, there is no plan for interim analyses of primary and/or secondary variables. 


\section{METHODS: MONITORING}

\section{Data monitoring}

There is no data monitoring committee for this study; several previous studies have already demonstrated that RBT is safe for people with stroke, with few adverse events reported. $^{12161920}$ Adverse events that meet all three of the following criteria will be reported immediately to the institution's Research Ethics Board, as is routine practice: (1) unexpected in terms of nature, severity or frequency; (2) related or possibly related to participation in the research; and (3) suggests a potential increase in risk of harm to research participants or others. All adverse events will be collated and evaluated biannually by the principal investigator.

\section{Potential harms}

In a previous study, mild adverse events related to RBT in people with stroke were delayed-onset muscle soreness, fatigue or exacerbation of joint pain $(11 \%, 7 \%$ and $32 \%$ of participants, respectively), ${ }^{19}$ which did not require medical attention, but resulted in reduced intervention intensity until they resolved (typically by the following session). Of note, the frequency and severity of adverse events are similar for the RBT group and control group, who completed more 'traditional' balance training. ${ }^{19}$ Therefore, these types of adverse events are typical of similar exercise programmes, and not specific to RBT.

As the assessment and intervention includes tasks that are deliberately challenging to balance control, there is a small risk that participants, on loss of balance, will fall. Appropriate precautions will be taken to ensure patient safety during these tasks. Interventions will be administered by a trained and licensed physiotherapist who will tailor the training to the patient's abilities. Assessments will be completed by a trained research assistant with a health sciences background. A safety harness attached to a secure point overhead will be worn for all postural perturbations to prevent a fall to the floor if the individual fails to regain stability. Additionally, the research assistant or physiotherapist can provide assistance to prevent a fall. We have administered tens of thousands of postural perturbations to over 500 individuals with varying balance abilities in previous research studies and clinical activities and no participant suffered an injury as a result of an induced postural perturbation. However, even if the participant is caught by the safety harness or researcher, there is a very small chance that participants will suffer a physical injury (eg, sprain or bruise). In the event of a minor physical injury, the physiotherapist will provide first aid, will advise the participant regarding follow-up with a medical professional (eg, family doctor) and home treatment (eg, rest, ice, compression, elevation) and will follow-up with the participant after a day or two.

The physiotherapist will communicate regularly with the participant's care team about changes in health status that could affect risk profile. Participants will be withdrawn if their health changes such that they would no longer be eligible for the study (ie, one of the exclusion criteria applies to them).

\section{ETHICS AND DISSEMINATION}

\section{Research ethics approval}

Research ethics approval has been received by the Research Ethics Board of the University Health Network (Study ID: 19-6001, approved 17 January 2020).

\section{Protocol amendments}

Substantive changes to the design or conduct of the study will require a formal amendment to the study protocol. Such substantive amendments will be agreed on by the study investigators and will be approved by the Research Ethics Board of the University Health Network prior to implementation. Minor administrative changes to study documents (eg, correcting a typographical error or clarifying a questionnaire item) may also be implemented, with the Research Ethics Board notified of the changes.

\section{Consent}

Potentially eligible participants will be identified by the patients' primary treating physiotherapist. The physiotherapist will ask patients if they are interested in speaking with a research assistant regarding the study. If patients agree, they will be approached by a member of the research team (DJ, CD or a delegate acting on their behalf) who will explain the study and provide patients with the study information sheet and consent form (online supplementary appendix 1). Research personnel will answer the patient's questions about the study. Patients may discuss the study with their friends, family members or healthcare providers. Patients may take as long as necessary to decide if they wish to participate in the study; however, if a patient has not decided before they are discharged then we will assume they have declined participation. The informed consent process will be documented by research personnel.

\section{Confidentiality}

Personal information is any information that could identify participants. If participants agree to join this study, the following personal information will only be accessible to the research team, for contact purposes: name, telephone number, mailing address and email address (if provided). A number of steps will be taken to ensure protection of personal health information. All information collected during this study, including the participant's personal information, will be kept confidential and will not be shared with anyone outside the study unless required by law. Electronic data will be stored on secure servers for 10 years. After 10 years the data will be deleted from the servers. Electronic files containing patient names and contact information will be password protected, and will be stored separately from study data. Hard copies of files containing deidentified data will be stored in locked cabinets and/or in offices that are locked when not occupied. 
Consent forms will be stored in locked cabinets/offices separately from other data. Only those individuals who require access to the data for the purpose of this study will be provided with the password to the file containing identifiers and/or the keys to the locked cabinet/office.

\section{Declaration of interests}

The authors declare that they have no competing interests related to this study.

\section{Access to data}

The principal investigator (AM) will have access to the full dataset. There is no current plan to make the participantlevel dataset available publicly; however, the dataset may be made available in future via a Data Access Committee, if such a committee is established by the institution.

\section{Ancillary and post-trial care}

The University Health Network will be responsible for providing out-of-pocket expenses to ensure that a participant receives immediate medical care in the event that the participant experiences an adverse health event (eg, injury) as a result of participation in the study. Patients do not typically receive follow-up after discharge from rehabilitation; therefore, there is no plan for any posttrial care.

\section{Dissemination policy}

Participants will receive a letter of appreciation at the end of the study, which may include a brief summary of the study results. Study results will be shared with the academic community via publication in peer-reviewed journals and presentations at conferences. We will aim to submit a paper describing analysis of the primary and secondary outcomes within 6 months of completing data collection. All individuals who meet the International Committee of Medical Journal Editors criteria for authorship will be included as authors on any publications arising from this work. We will share results directly with physiotherapists through interactive workshops (eg, at the Canadian Physiotherapy Association meeting). We are developing a toolkit to assist physiotherapists implementing RBT. The results of the larger trial will be incorporated into the toolkit as recommendations for RBT dose in subacute stroke.

\section{SIGNIFICANCE}

A high rate of falling is common after stroke, and fall risk is highest in the first months postdischarge from rehabilitation. ${ }^{23}$ RBT is a novel type of exercise that aims to improve reactive balance control, rather than 'traditional' balance training, which focuses on maintaining stability during voluntary movement. Time in stroke rehabilitation is limited, and physiotherapists' report on lack of time is a barrier to implementing RBT. ${ }^{41}$ The results of the proposed study will inform the design of a larger RCT to establish the optimal dose of RBT in subacute stroke. If a low dose of RBT can improve reactive balance control and prevent falls poststroke, this would allow therapists and patients to more easily include this fall-prevention intervention in rehabilitation, without sacrificing time spent working on other important rehabilitation goals.

\section{Twitter Avril Mansfield @avrilmansfield}

Contributors AM conceived the study and drafted the manuscript, and is the grant holder of this study. AM, ELI and CJD developed the intervention. AM, ELI, CJD, DJ, TB and AHH contributed to study design, writing/editing the manuscript and approved the final manuscript.

Funding This study is supported by the Heart and Stroke Foundation Canadian Partnership for Stroke Recovery. AM holds a New Investigator Award from the Canadian Institutes of Health Research (MSH-141983). We also acknowledge the support of the Toronto Rehabilitation Institute; equipment and space have been funded with grants from the Canada Foundation for Innovation, Ontario Innovation Trust and the Ministry of Research and Innovation. These funding sources had no role in the design of this study and will not have any role during its execution, analysis, interpretation of the data or decision to submit results.

Competing interests None declared.

Patient and public involvement Patients and/or the public were not involved in the design, or conduct, or reporting or dissemination plans of this research.

Patient consent for publication Not required.

Provenance and peer review Not commissioned; externally peer reviewed.

Open access This is an open access article distributed in accordance with the Creative Commons Attribution Non Commercial (CC BY-NC 4.0) license, which permits others to distribute, remix, adapt, build upon this work non-commercially, and license their derivative works on different terms, provided the original work is properly cited, appropriate credit is given, any changes made indicated, and the use is non-commercial. See: http://creativecommons.org/licenses/by-nc/4.0/.

\section{ORCID iDs}

Avril Mansfield http://orcid.org/0000-0002-0396-5815

Elizabeth L Inness http://orcid.org/0000-0002-9217-4619

David Jagroop http://orcid.org/0000-0001-8764-6258

Andrew H Huntley http://orcid.org/0000-0001-9523-304X

\section{REFERENCES}

1 Batchelor FA, Mackintosh SF, Said CM, et al. Falls after stroke. Int J Stroke 2012;7:482-90.

2 Watanabe Y. Fear of falling among stroke survivors after discharge from inpatient rehabilitation. Int J Rehabil Res 2005;28:149-52.

3 Friedman SM, Munoz B, West SK, et al. Falls and fear of falling: which comes first? A longitudinal prediction model suggests strategies for primary and secondary prevention. J Am Geriatr Soc 2002;50:1329-35.

4 Yardley L, Smith H. A prospective study of the relationship between feared consequences of falling and avoidance of activity in community-living older people. Gerontologist 2002;42:17-23.

5 Weerdesteyn V, de Niet M, van Duijnhoven HJR, et al. Falls in individuals with stroke. J Rehabil Res Dev 2008;45:1195-213.

6 Sherrington C, Tiedemann A, Fairhall N, et al. Exercise to prevent falls in older adults: an updated meta-analysis and best practice recommendations. N S W Public Health Bull 2011;22:78-83.

7 Batchelor F, Hill K, Mackintosh S, et al. What works in falls prevention after stroke?: a systematic review and meta-analysis. Stroke 2010;41:1715-22.

8 Verheyden GS, Weerdesteyn V, Pickering RM, et al. Interventions for preventing falls in people after stroke. Cochrane Database Syst Rev 2013;31:CD008728.

9 Mansfield A, Peters AL, Liu BA, et al. A perturbation-based balance training program for older adults: study protocol for a randomised controlled trial. BMC Geriatr 2007;7:12.

10 Mansfield A, Peters AL, Liu BA, et al. Effect of a perturbation-based balance training program on compensatory stepping and grasping reactions in older adults: a randomized controlled trial. Phys Ther 2010;90:476-91.

11 Mansfield A, Wong JS, Bryce J, et al. Does perturbation-based balance training prevent falls? systematic review and meta-analysis of preliminary randomized controlled trials. Phys Ther 2015;95:700-9. 
12 Mansfield A, Schinkel-Ivy A, Danells CJ, et al. Does perturbation training prevent falls after discharge from stroke rehabilitation? A prospective cohort study with historical control. J Stroke Cerebrovasc Dis 2017;26:2174-80.

13 Lohse KR, Lang CE, Boyd LA. Is more better? using metadata to explore dose-response relationships in stroke rehabilitation. Stroke 2014;45:2053-8.

14 Bhatt T, Yang F, Pai Y-C. Learning to resist gait-slip falls: long-term retention in community-dwelling older adults. Arch Phys Med Rehabil 2012;93:557-64.

15 Pai Y-C, Bhatt T, Yang F, et al. Perturbation training can reduce community-dwelling older adults' annual fall risk: a randomized controlled trial. J Gerontol A Biol Sci Med Sci 2014;69:1586-94.

16 Bhatt T, Dusane S, Patel P. Does severity of motor impairment affect reactive adaptation and fall-risk in chronic stroke survivors? J Neuroeng Rehabil 2019;16:43.

17 Lee A, Bhatt T, Liu X, et al. Can higher training practice dosage with treadmill slip-perturbation necessarily reduce risk of falls following overground slip? Gait Posture 2018;61:387-92.

18 Mansfield A, Inness EL, Mcllroy WE. Stroke. In: Day BL, Lord SR, eds. Handbook of clinical neurology: balance, gait, and falls. San Diego: Elsevier BV, 2018: 159. 205-28.

19 Mansfield A, Aqui A, Danells CJ, et al. Does perturbation-based balance training prevent falls among individuals with chronic stroke? a randomised controlled trial. BMJ Open 2018;8:e021510.

20 Schinkel-Ivy A, Huntley AH, Danells CJ, et al. Improvements in balance reaction impairments following reactive balance training in individuals with sub-acute stroke: a prospective cohort study with historical control. Top Stroke Rehabil 2020;27:262-71.

21 Handelzalts S, Kenner-Furman M, Gray G, et al. Effects of perturbation-based balance training in subacute persons with stroke: a randomized controlled trial. Neurorehabil Neural Repair 2019;33:213-24.

22 Bernhardt J, Hayward KS, Kwakkel G, et al. Agreed definitions and a shared vision for new standards in stroke recovery research: the stroke recovery and rehabilitation roundtable Taskforce. Int J Stroke 2017;12:444-50.

23 Forster A, Young J. Incidence and consequences of falls due to stroke: a systematic inquiry. BMJ 1995;311:83-6.

24 Chan A-W, Tetzlaff JM, Gøtzsche PC, et al. Spirit 2013 explanation and elaboration: guidance for protocols of clinical trials. BMJ 2013;346:e7586.

25 Mansfield A, Aqui A, Centen A, et al. Perturbation training to promote safe independent mobility post-stroke: study protocol for a randomized controlled trial. BMC Neurol 2015;15:87.

26 Raina P, Wolfson C, Kirkland S. Canadian longitudinal study on aging (CLSA) protocol, 2013. Available: https://clsa-elcv.ca/doc/511 [Accessed 22 Sep 2016].
27 Goldstein LB, Bertels C, Davis JN. Interrater reliability of the NIH stroke scale. Arch Neurol 1989;46:660-2.

28 Gowland C, Stratford P, Ward M, et al. Measuring physical impairment and disability with the Chedoke-McMaster stroke assessment. Stroke 1993;24:58-63.

29 Franchignoni F, Horak F, Godi M, et al. Using psychometric techniques to improve the balance evaluation systems test: the miniBESTest. J Rehabil Med 2010;42:323-31.

30 Powell LE, Myers AM. The Activities-specific balance confidence (ABC) scale. J Gerontol A Biol Sci Med Sci 1995;50A:M28-34.

31 Huntley AH, Rajachandrakumar R, Schinkel-lvy A, et al. Characterizing slip-like responses during gait using an entire support surface perturbation: comparisons to previously established slip methods. Gait Posture 2019;69:130-5.

32 König M, Epro G, Seeley J, et al. Retention of improvement in gait stability over 14 weeks due to trip-perturbation training is dependent on perturbation dose. J Biomech 2019;84:243-6.

33 Bhatt T, Pai Y-C. Prevention of slip-related backward balance loss: the effect of session intensity and frequency on long-term retention. Arch Phys Med Rehabil 2009;90:34-42.

34 Bhatt T, Wening JD, Pai Y-C. Influence of gait speed on stability: recovery from anterior slips and compensatory stepping. Gait Posture 2005;21:146-56.

35 Hyndman D, Ashburn A, Stack E. Fall events among people with stroke living in the community: circumstances of falls and characteristics of fallers. Arch Phys Med Rehabil 2002;83:165-70.

36 Myers AH, Baker SP, Van Natta ML, et al. Risk factors associated with falls and injuries among elderly institutionalized persons. Am J Epidemiol 1991;133:1179-90.

37 van der Ploeg HP, Streppel KRM, van der Beek AJ, et al. The physical activity scale for individuals with physical disabilities: test-retest reliability and comparison with an accelerometer. J Phys Act Health 2007;4:96-100.

38 Julious SA. Sample size of 12 per group rule of thumb for a pilot study. Pharm Stat 2005;4:287-91.

39 Tang Y. Sample size estimation for negative binomial regression comparing rates of recurrent events with unequal follow-up time. $J$ Biopharm Stat 2015;25:1100-13.

40 Tsang CSL, Liao L-R, Chung RCK, et al. Psychometric properties of the Mini-Balance evaluation systems test (Mini-BESTest) in community-dwelling individuals with chronic stroke. Phys Ther 2013;93:1102-15.

41 Mansfield A, Danells CJ, Inness EL, et al. A survey of Canadian healthcare professionals' practices regarding reactive balance training. Physiother Theory Pract 2019:1-14.

42 Kersten P, Ashburn A, George S, et al. The subjective index for physical and social outcome (SIPSO) in stroke: investigation of its subscale structure. BMC Neurol 2010;10:26. 